\title{
An outline of Cosmology based on interpretation of the Johannine Prologue
}

\author{
Victor Christianto
}

\begin{abstract}
As we know there are two main paradigms concerning the origin of the Universe: the first is Big-Bang Theory, and the other is Creation paradigm. But those two main paradigms each have their problems, for instance Big Bang Theory assumes that the first explosion was triggered by chance alone, therefore it says that everything emerged out of vacuum fluctuation caused by pure statistical chance. By doing so, its proponents want to avoid the role of the Prime Mover (God). Of course there are also other propositions such as the Steady State theory or Cyclical universe, but they do not form the majority of people in the world.

On the other side, Creation Theory says that the Universe was created by God in 6x24 hours according to Genesis chapter 1, although a variation of this theory says that it is possible that God created the Universe in longer period of thousands of years or even billions of years. But such a proposition seems not supported by Biblical texts.

To overcome the weaknesses of those main paradigms, I will outline here another choice, namely that the Universe was created by Logos (Christ in His pre-existence). This is in accordance with the Prolegomena of the Gospel of John, which says that the Logos was there in the beginning (John 1:1). I describe 3 applications of the classical wave equation according to Shpenkov, i.e. hydrogen energy states, periodic table of elements, and planetary orbit distances. For sure, Shpenkov derived many more results beside these 3 phenomena as discussed in his 3 volume books and many papers, but these 3 phenomena are selected to give clear examples of what can be done with the classical wave equation. And then I extend further the classical wave equation to fractal vibrating string.

While of course this outline is not complete, this article is written to stimulate further investigations in this direction.
\end{abstract}

\section{Introduction}

"In the beginning was the Word...," says the Gospel of John 1:1. (German: "Im Anfang war das Wort”. Greek: En avrch/| h=n o` lo,goj). This famous Prolegomena of the Gospel of John may be interpreted that everything comes from the Word of God, and since Word means Voice, and Voice means sound, and sound can be related to wave, vibration and frequency, then it seems quite straightforward to think that everything in this universe consists of vibration and frequency too.

While the above analogy with the Gospel of John is suggested by this writer, such a view that everything is related to wave and frequency has been proposed by George Shpenkov [1]. He wrote as follows: "A new physics paradigm that we have accepted and follow in all our works is based on: (1) Dialectical philosophy and dialectical logic; (2) The postulate on the wave nature of all phenomena and objects in the Universe.’[1, p.7]

Shpenkov uses the classical wave equation as follows:

$$
\Delta \hat{\Psi}^{\prime}-\frac{1}{c^{2}} \frac{\partial^{2} \hat{\Psi}^{\prime}}{\partial t^{2}}=0
$$

This wave equation is also known as the wave equation of sound. [2, p.12][3, p.111] In this paper we will discuss an outline of cosmology based on the proposed connection between vibrating string and the Logos in Prolegomena of the Gospel of John. We will also discuss some Shpenkov's achievements using the vibrating string (classical wave equation) model, such as hydrogen energy levels, periodic table of elements and also planetary orbits prediction. Then we will extend his model to become fractal vibrating string. 


\section{From Logos to Vibrating String}

In the context of scientific theories, we should admit that initially Big Bang Theory was made as a result of backward extrapolation of the Hubble law. The Hubble law itself only asserts that galaxies move away from each other. And if this law was extrapolated back to the origin of time, then we find that there should be a singularity which then was called as Big Bang. Many physicists have tried to explain what happened in the first minutes of the Big Bang, but so far no one can explain who triggered the Big Bang. Some physicists suggest that the Big Bang occur by chance alone out of vacuum fluctuation. ${ }^{1}$ It would mean that there is no Prime Mover of that Big Bang, except probabilistic chance. Another theory was proposed by Hawking; it is called the no boundary proposal, which means that the Universe does not need a Creator or God.

In other words, although at a first glance the Big Bang Theory is able to explain many astronomical data so far, it cannot give an answer to the philosophical question concerning who triggered the creation process in the beginning. Many physicists tried to avoid this penetrating problem. Therefore it seems that there is an open problem on how to reconcile Biblical answers with scientific explanation concerning the beginning of the Universe.

This writer would like to propose an interpretation i.e. if Genesis 1:1-2 is interpreted according to John 1:1, then it seems we can arrive at a different picture of creation, that is the Universe was created by the Word of God (Greek: Logos, Aramaic: Memra) with the power of the Spirit of God. ${ }^{2}$ And because the Logos is word, then it could mean voice or sound, and if sound can be interpreted as wave and frequency, then it seems quite logical to think that everything in the Universe are formed of wave and frequency (vibration). Therefore it is important to work on classical wave equation (vibrating string) instead of Schrödinger equation to model wave nature of atoms and molecules, partly because the wave mechanics is unrealistic model. ${ }^{3}$

A theory which supports this hypothesis is George Shpenkov's interpretation on the classical wave equation, which leads to the following conjectures: a. shell-nodal model of atoms and molecules; b. a periodic table of elements which is close to periodic table of Mendeleyev. ${ }^{4}$ And this writer proposed a further step, i.e. to extend further the classical wave equation to become fractal vibrating string, as mentioned briefly in his recent paper. ${ }^{5}$ Philosophically speaking, the fractal vibrating string has similarities with string theory, because both of them are based on the same hypothesis that particles come out of frequency and vibration, although they also have major difference that is string theorists must work with 26 dimensions: "... the universe has a total of 26 dimensions in string theory, as opposed to the four dimensions it possesses under Einstein's special and general relativity theories". Another different is that so far string theory has no single prediction which can be compared with observation or experiment, while the proposed fractal vibrating string model is closer to our everyday's experience.

\section{Memra in Targum and Christology of Colossians}

According to the Bible, Davar of Jahweh (Word of God) has the creating power, for example it can give breathe of life in Ez. 37:4-5, and it has role during creation of the heavens in Ps. 33:6.

Ezekiel 37:4-5 $\rightarrow$ "Again He said unto me, Prophesy upon these bones, and say unto them, O ye dry bones, hear the word of the LORD. Thus saith the Lord GOD unto these bones; Behold, I will cause breath to enter into you, and ye shall live:" (KJV)

\footnotetext{
${ }^{1}$ Bob Goette, Why talk about Creation?, Bible and Spade 03:2 (Spring 1990): 45-48

${ }^{2}$ Wilf Hildebrandt, An Old Testament Theology of the Spirit of God (Peabody, Massachusetts: Hendrickson Publishers, Inc., 1995), 29.

${ }^{3}$ Victor Christianto, A review on Schrödinger equation \& classical wave equation. Prespacetime Journal Vol.5 No. 5 , April 2014. URL: http://www.prespacetime.com or http://www.vixra.org/author/Victor_Christianto

${ }^{4}$ George Shpenkov, Schrödinger's error in principle. Galilean Electrodynamics, 2002. URL:

http://shpenkov.janmax.com

5 Victor Christianto, A derivation of GravitoElectroMagnetic (GEM) Proca-type equations in fractional space. Prespacetime Journal Vol. 5 No. 5, April 2014. URL: http://www.prespacetime.com or

${ }^{6}$ Andrew Zimmerman Jones \& Daniel Robbins, String Theory for Dummies (Indianapolis, Indiana: Wiley Publishing Inc., 2010), 169
} 
Psalm 33:6 $\rightarrow$ "By the word of the LORD were the heavens made; and all the host of them by the breath of His mouth." (KJV)

The above Ps. 33:6 has parallel with the Christology in Col. 1:16.

Colossians 1:16 $\rightarrow$ "For by Him were all things created, that are in heaven, and that are in earth, visible and invisible, whether they be thrones, or dominions, or principalities, or powers: all things were created by Him, and for Him." (KJV)

Therefore, we can conclude that everything in the Universe was created by the Logos or Christ Himself in His pre-existence. The name Word of God for Jesus Christ is also the same with the Kalimatullah title for Isa in Qur'an.

And the phrase the Logos is with God has also parallel in Targum of Gen. 31:24. See the following table:

Table 1. Comparison of some texts in Targum and Gospel of John

\begin{tabular}{|l|l|}
\hline Targum & Gospel of John \\
\hline Memra min qedem Alaha (Gen. 31:24) & Kai ho logos en pros ton theon (John 1:1b) \\
\hline Memra de Alaha (Gen. 19:17) & John. 1:3c \\
\hline
\end{tabular}

Therefore we can conclude that there are similarity of the concept of Word of God and His role in creation from John 1:1, Ps. 33:6, and Col. 1:16.

Since theological discussions on this issue can be very deep, I will only cite several papers for further reading by interested readers [10]-[16].

\section{The Classical Wave Equation and Hydrogen Energy States}

In his third book, ${ }^{7}$ George Shpenkov explains in detail on how to derive hydrogen energy states from the Classical wave equation above (1). In this section, I give a summary of his procedure.

According to Shpenkov, the hydrogen atom represents a simplest binary proton-electron system. According to the Dynamic Model (DM), which is the wave theory of micro-objects of atomic and sub-atomic levels, the hydrogen atom is the wave system of the longitudinal-transversal structure. It is a stable wave formation of the binary spherical-cylindrical wave field.

Spherical and cylindrical wave functions satisfying the wave equation (1) have, respectively, the following form:

$$
\hat{\Psi}^{\prime}=\hat{R}_{l}(k r) \Theta_{l-m}(\theta) \hat{\Phi}_{m}(\varphi) \hat{T}(\omega t),
$$

And

$$
\hat{\Psi}^{\prime}=\hat{R}_{m}\left(k_{r} r\right) \hat{Z}\left(k_{z} z\right) \hat{\Phi}_{m}(\varphi) \hat{T}(\omega t) .
$$

Radial components of spherical and cylindrical functions (2) and (3), respectively, are uniquely determined by the general structure of the following radial equations:

$$
\rho^{2} \frac{d^{2} \hat{R}_{l}}{d \rho^{2}}+2 \rho \frac{d \hat{R}_{l}}{d \rho}+\left(\rho^{2}-l(l+1)\right) \hat{R}_{l}=0
$$

And

$$
\frac{d^{2} \hat{R}}{d\left(k_{r} r\right)^{2}}+\frac{1}{k_{r} r} \frac{d \hat{R}}{d\left(k_{r} r\right)}+\left(1-\frac{m^{2}}{\left(k_{r} r\right)}\right) \hat{R}=0
$$

Where $\rho=k r$.

In the central spherical wave field of the hydrogen atom, amplitude of radial oscillations of the spherical shell of the proton, originated from solutions of (4), has the form:

$$
A_{s p h}=\frac{A \hat{e}_{1}(k r)}{k r},
$$

\footnotetext{
${ }^{7}$ George P. Shpenkov, Dialectical View of the World: Vol. 3 Dynamic Model of Elementary Particles - Part 2: Fundamentals. 2014. URL: http://shpenkov.janmax.com/Vol.3.DynamicModel-2.pdf
} 
Where

$$
\begin{aligned}
& \hat{e}_{l}(k r)=\sqrt{\frac{\pi k r}{2}}\left(J_{l+1 / 2}(k r) \pm Y_{l+1 / 2}(k r)\right), \\
& k=\frac{\omega}{c} .
\end{aligned}
$$

Here $\mathrm{J}(\mathrm{kr})$ and $\mathrm{Y}(\mathrm{kr})$ are Bessel functions; wis the oscillation frequency of pulsating spherical shell of the proton equal to the fundamental "carrier" frequency of the subatomic and atomic levels.

In the cylindrical wave field, the energy $E_{c y l}$, as the sum of energies of two mutually perpendicular potential-kinetic oscillations of the orbiting electron, is equal (to the simplest case) to:

$$
E_{c y l}=h v,
$$

Where

$$
h=\frac{2 \pi m_{e} c a^{2}}{r}=2 \pi m_{e} v A_{c y l},
$$

And

$$
A_{c y l}=\frac{a}{\sqrt{k r}} .
$$

Since the steady equilibrium exchange (interaction) between spherical and cylindrical fields in the hydrogen atom takes place invariably, the following equality is always valid:

$$
E_{c y l=-} E_{s p h}
$$

The above equation yields an equation which under condition of $\mathrm{p}=\mathrm{q}=0$ will yield the well-known elementary spectral formula of the hydrogen atom:

$$
\frac{1}{\lambda}=R\left(\frac{1}{m^{2}}-\frac{1}{n^{2}}\right)
$$

Where $\mathrm{m}$ and $\mathrm{n}$ are integers, and

$$
R=\frac{m_{0} c A^{2}}{2 h r_{0}^{2}}
$$

Is the Rydberg constant.

Thus, equations (13) and (14) complete Shpenkov's derivation of hydrogen energy states from the classical wave equation.

\section{The Classical Wave Equation and Periodic Table of Elements}

In his 2006 paper, Shpenkov explains a derivation from the classical wave equation to a periodic table of elements which is close to Mendeleyev's periodic table. ${ }^{8}$ In this section I will briefly summarize his results for convenience.

According to Shpenkov, one of the particular solutions of the 3-dimensional wave equation yields sinusoidal spherical standing waves described by Bessel functions. They are reminiscent of spherical resonant cavities having internal oscillating electric and magnetic mode fields. Their nodal structure uniquely determines the structure of matter at the atomic and molecular levels, in particular, the intra-atomic structure. On the basis of these solutions, the nature of the Periodic Law and symmetries of crystals are elucidated from a new point of view. This is based on interpretation of $\Psi$-function in equation (1) as the density of the potential-kinetic phase probability for the occurrence of events in the wave space.

As we know, equation (1) admits particular solutions of the form:

\footnotetext{
${ }^{8}$ George P. Shpenkov, “An Elucidation of the Nature of the Periodic Law," Chapter 7 in "The Mathematics of the Periodic Table", Rouvray, D. H. and King, R. B., ed. (New York: Nova Science Publishers, 2006), 119-160.
} 


$$
\hat{\Psi}(r, t)=\hat{\psi}(r) e^{ \pm i \omega t}
$$

Where $\omega=k c$ and $\hat{\psi}(r)$ is the particular solution of the corresponding Helmholtz equation

$$
\Delta \hat{\psi}+k^{2} \hat{\psi}=0
$$

The longitudinal component of the spherical-cylindrical field is described over a spherical realization of the wave equation (1). The separation of variables ${ }^{9}$ leads to one time equation :

$$
\frac{d^{2} \hat{T}}{d \tau^{2}}=-\hat{T},
$$

And three equations in spherical space :

$$
\begin{aligned}
& \rho^{2} \frac{d^{2} \hat{R}_{\ell}}{d \rho^{2}}+2 \rho \frac{d \hat{R}_{\ell}}{d \rho}+\left(\rho^{2}-\ell(\ell+1)\right) \hat{R}_{\ell}=0, \\
& \frac{d^{2} \Theta_{\ell, m}}{d \theta^{2}}+\operatorname{ctg} \theta \frac{d \Theta_{\ell, m}}{d \theta}+\left(\ell(\ell+1)-\frac{m^{2}}{\sin ^{2} \theta}\right) \Theta_{\ell, m}=0, \\
& \frac{d^{2} \hat{\Phi}_{m}}{d \varphi^{2}}+m^{2} \hat{\Phi}_{m}=0,
\end{aligned}
$$

Where $\rho=k r$ and $\tau=\omega t$.

By solving the above equations which involving the use of Bessel functions, Shpenkov arrives at a periodic table of elements which are close to Mendeleyev's periodic law. The result is shown in Figure 1 below.

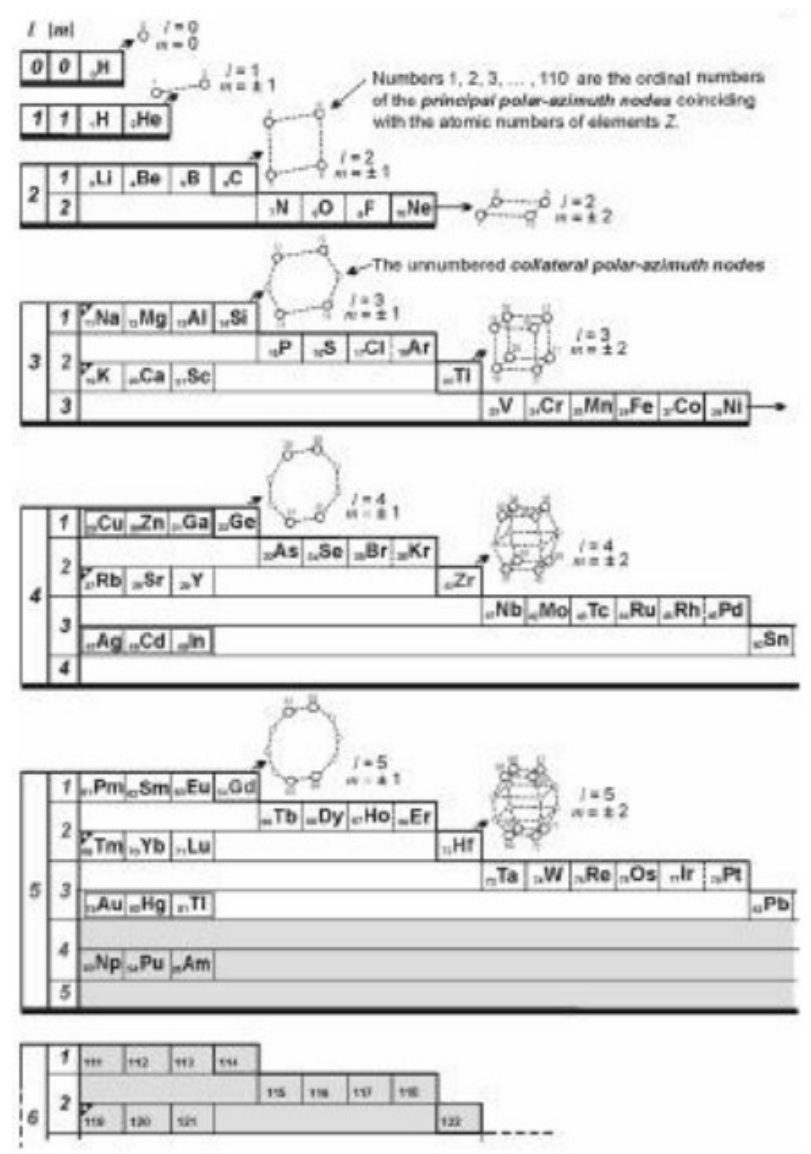

\footnotetext{
${ }^{9}$ For more discussion on separation of variables, see for example Karl Svozil, Mathematical Methods of Theoretical Physics, arXiv:1203.4558v4 [math-ph], 25 Mar. 2014, p. 203-206
} 


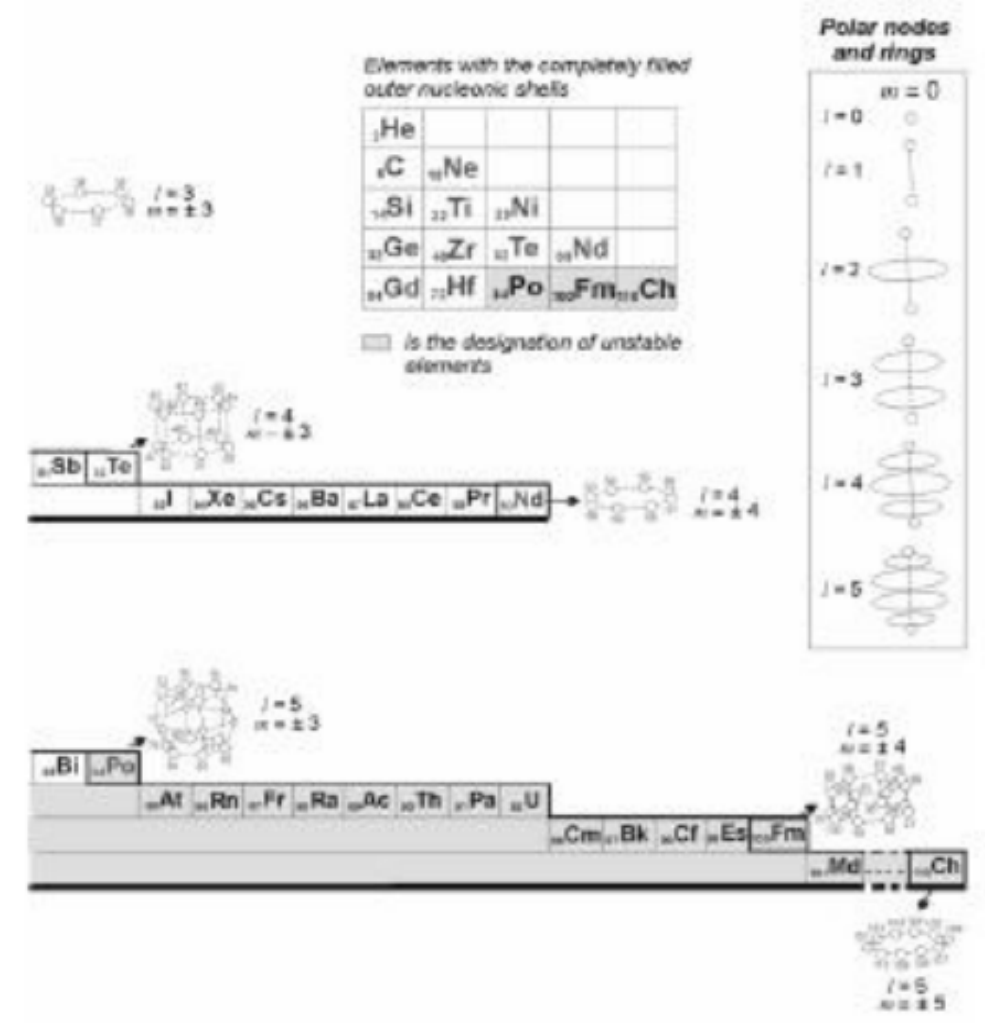

Figure 1. Generalized table of the elements from the particular solutions of the wave probabilistic equation (1), or the quasi-periodicity as a result of quasi-similarity of the nodal structure of external atomic shells. ${ }^{10}$

I will not repeat here Shpenkov's derivation, but those who are interested can consult his paper [18].

\section{The Classical Wave Equation and the Planetary Orbit Distances}

In one of his papers, Shpenkov derived planetary orbit distances from the same classical wave equation (1). ${ }^{11}$ In his interpretation, the gravitational frequency determines the gravitational radius of elementary particles, which is also the elementary radial gravitational wave:

$$
\hbar_{g}=\frac{c}{\omega_{g}}=3.274 \times 10^{11} \mathrm{~m}=327.4 \mathrm{Mkm}
$$

The wave shell of the gravitational radius of a particle in stellar systems, which in turn are spherical objects of mega space (atoms of mega world), separates the oscillating region of a spherical field-space of a star and its wave region.

In accordance with the solutions of the wave equation (1), the gravitational wave radius (21) of elementary particles determines the radii of their wave equilibrium spherical shells by the following equation:

$$
r=\lambda_{g} \cdot z_{m, n}=327.4 \times 10^{6} \times z_{m, n} \mathrm{~km},
$$

The solution (22) is realized in the first approximation of in a spectrum of the Keplerian shellsorbits, assuming that the gravitational shells are spherical and, therefore, the orbits are circular. Under the conditions of interplanetary gravitation interaction, the planets cannot move strictly along circular orbits, to which they naturally aspire constantly as to equilibrium. Mutual perturbations eventually have turned the circular orbits in elliptic.

\footnotetext{
${ }^{10}$ Shpenkov, "An Elucidation of the Nature of the Periodic Law," 144-145.

${ }^{11}$ George Shpenkov, "Planetary Orbits," Chapter 6 in Some words about Fundamental Problems of Physics, 2011. URL: http://shpenkov.janmax.com
} 
According to Shpenkov, if we take as the basic, a gravitational wave shell of the Sun, e.g. on which is an orbit of the planet Mercury, we arrive at the gravitational spectrum, conditioned by the solutions of the Bessel functions of the first order (see Table 2)

Table 2. A gravitational spectrum of wave spherical shells of elementary particles ${ }^{12}$

\begin{tabular}{|l|l|l|l|}
\hline $\mathrm{S}$ & $z_{m, n}=j_{1, s}$ & $\mathrm{r}_{\mathrm{s}}, \mathrm{Mkm}$ & Planets \\
\hline 1 & 3.831706 & 57.91 & Mercury \\
\hline 2 & 7.015587 & $106.03(108.2)$ & Venus \\
\hline 3 & 10.17347 & $153.76(149.6)$ & Earth \\
\hline 4 & 13.32369 & $201.36(204.5)$ & Toro \\
\hline 5 & 16.47063 & $248.93(227.9)$ & Mars \\
\hline 6 & 19.61586 & 296.46 & Asteroids \\
\hline 7 & 22.76008 & 339.45 & Asteroids \\
\hline 8 & 25.90367 & 391.49 & Asteroids \\
\hline 9 & 29.04683 & 438.96 & $413.77(1$ Ceres) \\
\hline 10 & 32.18968 & 486.49 & Asteroids \\
\hline 11 & 35.33231 & 533.99 & Asteroids \\
\hline 12 & 38.47476 & 581.48 & Asteroids \\
\hline 13 & 41.61709 & 628.97 & 1 Asteroid \\
\hline 14 & 44.75932 & 676.46 & \\
\hline 15 & 47.90146 & 723.95 & \\
\hline 16 & 51.04354 & $771.44(778.57)$ & Jupiter \\
\hline 30 & 95.02923 & $1436.2(1433.45)$ & Saturn \\
\hline
\end{tabular}

Note: semi-major axes of elliptical orbits of the planets are in brackets. For a small planet Toro, in brackets, an average distance from the Sun is indicated.

Beside the above result, Shpenkov also derives orbits of satellites of Jupiter, Saturn, and Uranus.

7. Possible Extensions from The Classical Wave Equation in Fractal Vibrating String

In this section I will give an outline of wave equation in fractal vibrating string and also wave equation on Cantor sets. These equations can be used to extend the classical wave equation discussed in the previous sections.

a. The wave equation in fractal vibrating string

$\mathrm{Hu}$, Agarwal \& Yang [21] introduce a local fractional wave equation in fractal vibrating string which is described as:

$$
\frac{\partial^{2 \alpha} u(x, t)}{\partial t^{2 \alpha}}+a^{2 \alpha} \frac{\partial^{2 \alpha} u(x, t)}{\partial x^{2 \alpha}}=0,
$$

With some fractal boundary conditions as defined in [21, p. 2].

Now we look at the particular solutions of the form:

$$
u(x, t)=\phi(x) T(t),
$$

And arrive at the equations:

$$
\begin{gathered}
\phi^{(2 \alpha)}+\lambda^{2 \alpha} \phi=0, \\
T^{(2 \alpha)}+a^{2 \alpha} \lambda^{2 \alpha} T=0 .
\end{gathered}
$$

\footnotetext{
${ }^{12}$ Ibid, p.40
} 
b. The 3-D wave equation on Cantor sets

According to Su, Yang, Jafari \& Baleanu [22], 3-D wave equation on Cantor sets described by the local fractional derivative can be written as follows:

$$
\frac{\partial^{2 \alpha} u(x, y, z, t)}{\partial t^{2 \alpha}}+a^{2 \alpha} \nabla^{2 \alpha} u(x, y, z, t)=0
$$

Where local fractional Laplace operator is noted by:

$$
\nabla^{2 \alpha}=\frac{\partial^{2 \alpha}}{\partial x^{2 \alpha}}+\frac{\partial^{2 \alpha}}{\partial y^{2 \alpha}}+\frac{\partial^{2 \alpha}}{\partial z^{2 \alpha}} \text {. }
$$

c. Solution of the Wave equation on Cantor sets using Local Fractional Series Expansion Method Yang, Yang \& Li [23] describes a new method to solve the wave equation on Cantor sets. The wave equation on Cantor sets is given by:

$$
\frac{\partial^{2 \alpha} u(x, t)}{\partial t^{2 \alpha}}+c \frac{\partial^{2 \alpha} u(x, t)}{\partial x^{2 \alpha}}=0
$$

Where $\mathrm{c}$ is a constant and $0<\alpha \leq 1$. The initial condition is:

$$
u(x, 0)=E_{\alpha}\left(x^{\alpha}\right) .
$$

Then they obtain the solution as follows [23]:

$$
u(x, t)=E_{\alpha}\left(x^{\alpha}\right)\left[\cosh _{\alpha}\left(c t^{\alpha}\right)+\sinh _{\alpha}\left(c t^{\alpha}\right)\right] \text {. }
$$

The above three methods may be applied for cosmological and astrophysical problems.

\section{Some Implications and Concluding Remarks}

I outlined here a new choice for cosmology theory, namely that the Universe was created by Logos (Christ in His pre-existence). This is in accordance with the Prolegomena of the Gospel of John, which says that the Logos was there in the beginning (John 1:1).

I describe 3 applications of the classical wave equation according to Shpenkov, i.e. hydrogen energy states, periodic table of elements, and planetary orbit distances. For sure, Shpenkov derived many more results beside these 3 phenomena as discussed in his 3 volume books and many papers, but these 3 phenomena are selected to give clear examples of what can be done with the classical wave equation.

And then I extend further the classical wave equation to fractal vibrating string. I describe three different approaches to extend the classical wave equation. It can be expected that these approaches may be applied for cosmological and astrophysical problems.

While of course this outline is not complete, this article is written to stimulate further investigations in this direction.

\section{References:}

[1] Shpenkov, George P. 2013. Dialectical View of the World: The Wave Model (Selected Lectures). Volume I: Philosophical and Mathematical Background. URL:

http://shpenkov.janmax.com/Vol.1.Dialectics.pdf

[2] Rienstra, S.W., \& Hirschberg, A. 2014. An Introduction to Acoustics. Eindhoven University of Technology. URL: www.win.tue.nl/ sjoerdr/papers/boek.pdf

[3] Pain, H.J. 2005. The Physics of Vibrations and Waves. 6th ed. John Wiley \& Sons, Ltd. ISBN: 0-470-01295-1(hardback); 0-470-01296-X(paperback). 563 pp.

[4] Goette, Bob. 1990. Why talk about Creation?, Bible and Spade 03:2 (Spring 1990): 45-48

[5] Hildebrandt, Wilf. 1995. An Old Testament Theology of the Spirit of God. Peabody, Massachusetts: Hendrickson Publishers, Inc., 29; see also [5a] Waltke, Bruce K. 1975. The Creation Account in the Genesis 1:1-3, Part IV: The theology of Genesis 1, Bibliotheca Sacra 132:528 (Oct. 1975): 327-341. URL: http://www.galaxie.com/journals 
[6] Shpenkov, George. 2002. Schrodinger's error in principle. Galilean Electrodynamics. URL: http://shpenkov.janmax.com

[7] Christianto, Victor. 2014. A review on Schrödinger equation \& classical wave equation. Prespacetime Journal Vol.5 No. 5, April 2014. URL: http://www.prespacetime.com or http://www.vixra.org/author/Victor_Christianto

[8] Christianto, Victor. 2014. A derivation of GravitoElectroMagnetic (GEM) Proca-type equations in fractional space. Prespacetime Journal Vol. 5 No. 5, April 2014. URL:

http://www.prespacetime.com or http://vixra.org/author/Victor_Christianto

[9] Zimmerman Jones, Andrew, \& Robbins, Daniel. 2010. String Theory for Dummies. Indianapolis, Indiana: Wiley Publishing Inc., p. 169.

[10] Parker, James. 1988. The Incarnational Theology of John. Criswell Theological Review 3.1, 31-48.

[11] Boyarin, Daniel. 2001. The Gospel of the Memra: Jewish Binitarianism and the Prologue to John. The Harvard Theological Review, Vol. 94 no. 3, pp. 243-284.

[12] Byrne, Brendan. 1997. Christ's pre-existence in Pauline Soteriology. Theological Studies 58, pp. 308-330.

[13] Helyer, Larry L. 1994. Cosmic Christology and Col. 1:15-20. JETS 37:2, pp. 236-246.

[14] Henebury, Paul Martin. 2004. Jesus Christ, The Logos of God: An inquiry into the Johannine Prologue and Its Significance. CTJ 08:23, pp. 88-105.

[15] Shepherd, Michael B. 2008. Targums, The New Testament, and Biblical Theology of the Messiah. JETS 51:1, pp. 46-58.

[16] Kim, Stephen S. 2009. The Literary and Theological Significance of the Johannine Prologue. BSAC 166:664, pp. 422-435.

[17] Shpenkov, George P. 2014. Dialectical View of the World: Vol. 3 Dynamic Model of Elementary Particles - Part 2: Fundamentals. http://shpenkov.janmax.com/Vol.3.DynamicModel2.pdf

[18] 2006. An Elucidation of the Nature of the Periodic Law, Chapter 7 in "The Mathematics of the Periodic Table", Rouvray, D. H. and King, R. B., ed., Nova Science Publishers, NY, pp. 119-160.

[19] Svozil, Karl. 2014. Mathematical Methods of Theoretical Physics. arXiv:1203.4558v4 [math$\mathrm{ph}]$

[20] Shpenkov, George P. 2011. "Planetary Orbits," Chapter 6 in Some words about Fundamental Problems of Physics. URL: http://shpenkov.janmax.com

[21] Hu, Ming-Sheng, Agarwal, Ravi P., \& Yang, Xiao-Jung. 2012. Local Fractional Fourier Series with Application to Wave equation in Fractal Vibrating String. Abstract and Applied Analysis Vol. 2013, Article ID. 567401, doi:10.1155/2012/567401

[22] Su, Wei-Hua, Yang, Xiao-Jun, Jafari, H., \& Baleanu, Dumitru. 2013. Fractional complex transform method for wave equations on Cantor sets within local fractional differential operator. Advances in Difference Equations 2013:97, http://www.advancesindifferenceequations.com/content/2013/1/97

[23] Yang, Ai-Min, Yang, Xiao-Jun, \& Li, Zheng-Biao. 2013. Local Fractional Series Solution Method for solving Wave and Diffusion Equations on Cantor Sets. Abstract and Applied Analysis Vol. 2013, Article ID. 351057, http://dx.doi.org/10.1155/2013/351057 